\title{
Breast Carcinoma with Melanotic Features
}

National Cancer Institute

\section{Source}

National Cancer Institute. Breast Carcinoma with Melanotic Features. NCI Thesaurus. Code C40351.

A very rare primary malignant tumor of the breast, characterized by an invasive breast carcinoma that co-exists with a melanoma component. The vast majority of melanotic tumors that affect the breast are metastatic melanomas that originate in extramammary sites. 